Article

\title{
Can Blockchain Help Resolve Modern Slavery in Supply Chains?
}

\author{
Martijn Boersma', Justine Nolan² \\ 1 University of Technology Sydney, Australia, ${ }^{2}$ University of New South Wales, Australia \\ Keywords: ethical sourcing, transparency, supply chains, blockchain, modern slavery \\ https://doi.org/10.46697/001c.13542
}

\section{AIB Insights}

Vol. 20, Issue 2, 2020

While blockchain was designed as a ledger for cryptocurrency transactions, it can record transactions of anything of value. Blockchain is increasingly used to prove the integrity of commodities, tracing their supply chain journey from the source to the end user. Yet, transferring this technology from a cryptocurrency context to a supply chain setting is not without difficulties. This article explores the implications for multinational and transnational companies in using blockchain as a means to address modern slavery. The research identifies five challenges: verification, inclusion, trust, privacy, and normativity.

\section{INTRODUCTION}

Blockchain can be used to create a record of transactions for anything of value. It is increasingly used to prove the integrity of commodities, tracing their supply chain journey from the source to the end user. For example, together with a Fijian fishing company and a technology company based in the United States, the World Wildlife Fund in Australia, Fiji and New Zealand piloted the use of blockchain to track tuna, showing when and where it was caught (Visser \& Hanich, 2018). Their aim was to verify that companies and consumers are selling or buying tuna that is sustainably caught and free of slave labor. As blockchain is widely touted as a means to address modern slavery, this article explores the implications for companies engaged in multinational and transnational activities.

\section{BLOCKCHAIN TECHNOLOGY AND MODERN SLAVERY}

Blockchain is best thought of as a public ledger, distributed through a peer-to-peer network where participants validate transactions and maintain a copy of the ledger. Changes to the ledger can only be made through agreement among blockchain participants. This process is underpinned by a consensus protocol, which determines who gets to validate a transaction. Decision makers could be all the blockchain participants, a majority, a select few, or a random selection. Once a transaction is validated, an irreversible record of that transaction is created. These records are called blocks and are chained together chronologically. While blockchain was designed as a ledger for cryptocurrency transactions, it can record transactions of anything of value.

In supply chains, blockchain can provide information about the provenance of goods. This can be beneficial to companies and their stakeholders, as the oversight of companies on the origin of goods in their supply chains is often limited, especially where it concerns cross-border sourcing. Because of this companies are at risk of being connected to modern slavery (Stringer \& Michailova, 2018). For example, the demand for cobalt - a mineral used in the production of phone and laptop batteries - has been met by people trapped in modern slavery (Frankel, 2016). Modern slavery includes forced labor (involuntary work under the threat of punishment); bonded labor (work to pay off a debt while losing control over conditions and repayments); trafficking (the movement of people for exploitation); and child slavery (exploitation of children for someone else's gain) (Nolan \& Boersma, 2019).

It is crucial to see modern slavery through the prism of broader labor rights. While some engage in work that from the outset involves adverse conditions, others engage in work with the expectation of decent conditions but find that circumstances worsen without avenues for exit. When abuses such as non-payment of wages and benefits, unfair dismissals, forced and unpaid overtime, and the denial of freedom of association and collective bargaining are prevalent and left unchecked, more severe forms of exploitation can develop (Nolan \& Boersma, 2019). Using blockchain, companies engaged in multinational and transnational activities may gain insight into the conditions in their supply chains and (the risk) of modern slavery. Yet, transferring this technology from a cryptocurrency context to a supply chain setting is not without difficulties. We identify five challenges for the use of blockchain to address modern slavery: verification, inclusion, trust, privacy and normativity.

\section{CHALLENGES FOR BLOCKCHAIN IN ADDRESSING MODERN SLAVERY}

\section{VERIFICATION}

Blockchain was designed to verify cryptocurrency transactions and provide transparency without the need for an intermediary such as a bank. In supply chains it can be used in a similar way, to verify commodity transactions and to provide transparency about labor standards without the need for an intermediary such as a social audit firm. To do this, every supply chain transaction needs to be recorded, especially since the visibility of working conditions decreases further upstream. Crucially, to address (the risk of) modern slavery it is not just the transaction that needs to be verified, the conditions surrounding transactions need to be validated at each step in the sourcing and production process. There is therefore a difference between a blockchain that shows the provenance of goods, and a blockchain that aims to show that a commodity is free of slave labor.

As the number of supply chain tiers increases, so will the 
number of transactions and blockchain participants. This is where establishing adequate conditions for consensus becomes challenging. The verification of a transaction is achieved via the consensus protocol, which can involve the input of all the blockchain participants, a majority, a select few or a random selection. While decentralized governance is a blockchain strength, it also carries risk. For example, in a cryptocurrency blockchain that requires a majority of actors to validate transactions, so-called 51\% attacks have been used to control the validation mechanism with ill intent (Rijmenam \& Ryan, 2018). In a supply chain, through majority rule, malevolent blockchain actors would be able to validate dubious transactions or choose not to scrutinize the conditions surrounding certain transactions.

\section{INCLUSION}

A solution is to use a private rather than a public blockchain. Both operate in a similar way, yet access to a private blockchain is invite-only. The actors that validate transactions can therefore be vetted. This negates two blockchain elements: it undermines transparency, and by excluding actors the democratic character of blockchain is weakened. Another solution is to make reputation a key determinant: a select few reputable actors are relied upon to validate a transaction. Yet, this too undermines the democratic character of blockchain, and relying on a select few actors means that they serve as intermediaries, which can reduce the efficiency of the system. It is also crucial that workers are involved in validating transactions, yet this is unlikely if they are being exploited.

While blockchain is decentralized and does not require an intermediary, its uptake to address modern slavery is likely to be driven by companies at the top of supply chains, which are most prone to reputational damage from being linked to exploitation. While these companies can wield their buyer leverage to get suppliers on board, their influence likely decreases as the number of supply chain tiers increases and suppliers become geographically dispersed. Ensuring blockchain uptake throughout the supply chain and across borders therefore becomes a cascading responsibility, where companies at each tier and in each location require their suppliers to use blockchain. Yet not everyone may be enticed to do so. In cryptocurrency blockchains, when a block is validated, validators are rewarded with cryptocurrency. In non-cryptocurrency blockchains, actors may lack an incentive to validate transactions and even the opposite can be true: suppliers may not want to use blockchain because of questionable labor practices that could be exposed. Furthermore, smaller suppliers (especially in developing countries) may lack the financial means or technological infrastructure to participate. The use of blockchain may therefore form a barrier to market entry and may inadvertently push workers (further) into the dark economy.

\section{TRUST}

One of blockchain's strengths is its ability to log transactions in a tamperproof distributed ledger. Yet, when verifying the integrity of goods in supply chains, this does not mean that the link between a block and its material reality should go unquestioned. While a block cannot be manipulated retrospectively, the integrity of information that is initially entered is vulnerable to inaccuracies or fraud. Forced labor generates US\$ 150 billion in illegal profits per year (International Labour Organization, 2014). There is therefore a risk that blockchain can be used to launder value that is illegally generated. Apart from the consensus protocol, there is no mechanism to stop actors from creating a block that claims that a commodity is free of slave labor, whether this is true or not.

That risk is pertinent as modern slavery is a complex issue. In order to verify transactions and rule out the possibility of modern slavery and other forms of exploitation, an adequate amount of information about working conditions needs to be included in the blockchain. There is a danger that the amount of information undermines the efficiency of the system, while the information may likewise be too complicated for other blockchain participants to comprehend. An intermediary could help to overcome this problem, but this negates one of the key advantages of using blockchain. Conversely, the inclusion of information that is too basic creates the risk that a complex matter such as modern slavery becomes oversimplified, and that the key factors underlying labor exploitation are ignored.

\section{PRIVACY}

The inclusion of large amounts of information can also create problems in terms of privacy. In a public blockchain, the fact that anyone can access the ledger would mean that the prices of goods and labor would no longer be confidential. For workers, the notion of transparency and immutable records can also be problematic: they could face repercussions if they refuse to validate a transaction because of labor standards concerns. Workers may also be branded as "difficult", as the immutability of blockchain makes it difficult for a person to be "forgotten". Coupled with encryption, blockchain could potentially handle sensitive information, however it would be difficult to verify if workers get paid appropriately if information on the prices of goods and labor is withheld, and anonymity may not offer workers protection from punitive measures in smaller workplaces.

Another way to solve this problem is by using a "hybrid blockchain", which combines elements of public and private blockchains (Sagirlar, Carminati, Ferrari, Sheehan, \& Ragnoli, 2018). Hybrid blockchains also validate and record transactions in a tamperproof ledger, but participation can be invite-only and on the basis of anonymity (the identity of participants is revealed to other parties in transactions, not to every blockchain participant), while participants can decide which transactions are made public. Hybrid blockchains can be designed to find a balance between inclusion, efficiency and transparency. However, this creates the risk that a hybrid blockchain favours efficiency, rather than to be inclusive and provide transparency. Put differently, hybrid blockchains can be configured to exclude certain actors and transactions, leading to a weakened protocol to validate transactions, while providing parties such as labor rights organisations with limited insight into supply chains.

\section{NORMATIVITY}

Addressing exploitation in supply chains is challenging, as the role of government as the traditional arbitrator of such issues has become less prominent. In addition, the increase in multinational and transnational business activities has resulted in governance gaps. As blockchain does not require an intermediary, it is ostensibly capable of grappling with the reduced role of government. Firms from institutionally distant environments often engage in partnerships in response to governance gaps (Abdi \& Aulakh, 2012). 
Blockchain seems to be well suited to facilitate such partnerships, as it allows stakeholders in institutionally distant environments to enforce the mutually agreed upon norms concerning the circumstances in which supply chain transactions are deemed to be acceptable.

Yet the standards that a blockchain upholds are not uncontentious. To address governance gaps, a sustainability standards market has emerged which has led to rival certification standards (Fransen \& Burgoon, 2012). Given the development of several ethical sourcing blockchains (Smith, 2019), it is possible that a similar market will emerge with competing blockchains. The existence of rival blockchain platforms could have normative implications, as platforms with comparatively weak standards could develop. It would also be a mistake to see blockchain as a mere facilitator of governance. Blockchain technology is not neutral: through its configuration it can be exclusive of actors and it can exhibit various levels of transparency.

\section{BLOCKCHAIN IS NOT A SILVER BULLET TO ADDRESS MODERN SLAVERY}

Hypothetically, blockchain offers a democratic system that gives control to a network of corporate stakeholders to verify transactions and working conditions in supply chains. However, to effectively address (the risk of) modern slavery, transactions at all supply chain tiers need to be verified, especially further upstream. The process to determine whether all transactions and the labor conditions surrounding them are acceptable poses a significant challenge. Achieving this through an unrestricted public blockchain, via majority rule, may not suit the needs of workers or companies as the incentives of a majority of blockchain actors may not always be aligned with the common good.

The capacity of workers to participate in the blockchain is vital. Unequal access undermines the legitimacy of the validation process, while a digital divide due to a lack of financial or technological means could (further) marginalize workers. Although the ledger is tamperproof, the link between a block and its material reality should not go unquestioned. As with any other technology, the saying "garbage in, garbage out" applies. Given the size of the dark economy, blockchain may be used to sanitize goods produced using slave labor. As modern slavery is a complex issue, an adequate amount of information about working conditions must be included in the blockchain. Yet, doing so may hinder its efficient functioning, while radical transparency also raises privacy concerns. Using alternatives such as private and hybrid blockchains involves a difficult compromise in terms of inclusion, efficiency and transparency, while the rise of competing ethical sourcing blockchains may result in the creation of weaker platforms.

Blockchain does not offer a silver bullet to address (the risk of) modern slavery. An effective response requires companies engaged in multinational and transnational business activities to consider the myriad of factors that lead to modern slavery, rather than to rely on a technological fix for a complex social problem. Failing to do so results in an incomplete understanding of the problem and reduces the likelihood of an effective response. Those teaching international business may wish to engage their students to see how they, as digital natives, view the role of blockchain to address labor exploitation in a global context. For international business researchers, the challenges identified in this paper may form the basis for further academic inquiry into the use of blockchain to address labor abuses.

\section{ABOUT THE AUTHORS}

Martijn Boersma (martijn.boersma@uts.edu.au) is a senior lecturer at the business school of the University of Technology Sydney. He is interested in the intersection of business and society. Current projects focus on multistakeholder efforts to improve working conditions in the Australian commercial real estate cleaning supply chain, and strategies to improve labor conditions within the Australian cotton value chain. Together with Justine Nolan he published the book Addressing Modern Slavery (2019).

Justine Nolan (justine.nolan@unsw.edu.au) is a Professor at UNSW Law and a Visiting Scholar at NYU Stern Center for Business and Human Rights. She examines the human rights impacts in global supply chains and more generally the intersection of business operations with human rights. Her most recent work is Addressing Modern Slavery (2019) with Martijn Boersma. 


\section{REFERENCES}

Abdi, M., \& Aulakh, P. S. 2012. Do country-level institutional frameworks and interfirm governance arrangements substitute or complement in international business relationships? Journal of International Business Studies, 43(5): 477-497.

Frankel, T. C. 2016, September 30. This is where your smartphone battery begins. Washington Post. http s://www.washingtonpost.com/graphics/business/batt eries/congo-cobalt-mining-for-lithium-ion-battery/.

Fransen, L., \& Burgoon, B. 2012. A market for worker rights: Explaining business support for international private labour regulation. Review of International Political Economy, 19(2): 236-266.

International Labour Organization. 2014, May 20. ILO says forced labour generates annual profits of US\$150 billion. http://www.ilo.org/global/about-the-ilo/news room/news/WCMS_243201/lang--en/index.htm.

Nolan, J., \& Boersma, M. 2019. Addressing Modern Slavery. Sydney: UNSW Press.
Rijmenam, M. V., \& Ryan, P. 2018. Blockchain. London; New York: Routledge.

Sagirlar, G., Carminati, B., Ferrari, E., Sheehan, J. D., \& Ragnoli, E. 2018. Hybrid-IoT: Hybrid Blockchain Architecture for Internet of Things - PoW Subblockchains. Presented at the 2018 IEEE Blockchain International Conference. https://doi.org/10.1109/ cybermatics_2018.2018.00189.

Smith, P. 2019. Ex-Qantas exec tackles food slavery with blockchain. Australian Financial Review. http s://www.afr.com/technology/ex-qantas-exec-tackles-f ood-slavery-with-blockchain-20190728-p52bgm.

Stringer, C., \& Michailova, S. 2018. Why modern slavery thrives in multinational corporations' global value chains. Multinational Business Review, 26(3): 194-206.

Visser, C., \& Hanich, Q. 2018. How blockchain is strengthening tuna traceability to combat illegal fishing. The Conversation. http://theconversation.co $\mathrm{m}$ /how-blockchain-is-strengthening-tuna-traceabilit y-to-combat-illegal-fishing-89965. 